\title{
Clima escolar, estilos de enfrentamiento del personal de apoyo y percepción estudiantil de violencia escolar
}

\section{School climate, coping styles of support personnel and students' perception of school violence}

DOI: https://doi.org/10.32870/dse.v0i24.1042

\author{
Jesús Tánori Quintana* \\ José Ángel Vera Noriega** \\ Gildardo Bautista Hernández*** \\ Francisco Fernando Durazo Salas****
}

\begin{abstract}
Resumen
La comunidad escolar, estudiantes, docentes, personal de apoyo y directivos, cada uno tiene un rol específico para generar una convivencia adecuada. El objetivo es identificar la relación entre la percepción del clima escolar, el tipo de enfrentamiento ante la violencia por parte del personal de apoyo académico (prefectos, trabajadora social y orientadores) y la percepción de violencia escolar en secundarias públicas del estado de Sonora, México. Participaron 154 personas quienes realizan trabajo de apoyo para la gestión directiva en 46 secundarias públicas de Sonora (46.6\% del sexo masculino y $53.4 \%$ del femenino). Los resultados señalan directamente que el enfrentamiento directo se relaciona positivamente con el compromiso y vinculación, y de manera negativa con el de tipo evitativo, mientras que los efectos indirectos señalan que el enfrentamiento directo se relaciona positivamente con el clima escolar positivo y que el enfrentamiento evasivo lo hace de manera negativa. Además, se muestra que el clima escolar se relaciona de manera positiva con la percepción de violencia. Los resultados indican que el papel que asume el personal de apoyo frente a la violencia escolar está marcado por su percepción del clima escolar y la forma en la que enfrenta las situaciones de acoso entre los estudiantes.
\end{abstract}

Palabras clave: enfrentamiento - enseñanza secundaria - gestión educacional - ambiente educacional - violencia.

\footnotetext{
* Doctor en Ciencias Sociales. Profesor interino en el Departamento de Educación, Instituto Tecnológico de Sonora, Ciudad Obregón, Sonora. México. jesus.tanori@itson.edu.mx

** Doctor de educación. SNI II. Miembro de la Academia Mexicana de la Ciencia. Líneas de investigación: desarrollo social, evaluación educativa, socialización escolar y calidad de vida en poblaciones vulnerables y de riesgo. Investigador Titular "E" del Centro de Investigación en Alimentación y Desarrollo A. C (CIADAC). Académico, Universidad de Sonora. México. (Autor por correspondencia). avera@ciad.mx

*** Doctor en Desarrollo Regional. Docente de tiempo completo de la Licenciatura en Enfermería, Universidad Intercultural del Estado de Puebla, México.gilbher@gmail.com

**** Licenciado en Psicología Industrial. Investigador asociado en consultora TECNOESTATA, S.C. México. durazof@gmail.com
} 


\begin{abstract}
Within the school community - students, teachers, support staff and directors - each element plays a specific role to generate an adequate coexistence. The aim of our research is to identify the relationship among the perception of the school climate, the ways of coping with violence of academic support personnel (prefects, social workers, and counselors) and the perception of school violence in public secondary schools in the state of Sonora, Mexico. 154 people who do support work for the directive management in 46 public secondary schools in Sonora ( $46.6 \%$ male and $53.4 \%$ female) took part in this study. The results indicate that dealing with violence directly is positively linked to commitment and bonding and avoidance is linked negatively, while the indirect effects indicate that dealing with violence directly is positively linked to a favorable school climate and evasive coping is linked negatively. Furthermore, we found that the school climate is positively linked to the perception of violence. Our results indicate that the role played by support personnel in the face of school violence is marked by their perception of the school climate and the way in which they deal with bullying situations among students.
\end{abstract}

Keywords: coping - secondary education - educational management - educational environment - violence.

\title{
Introducción
}

El análisis de la violencia en las escuelas requiere un enfoque que incluya a los diversos actores que participan en ella. En una comunidad escolar, cada integrante tiene un rol específico para generar una convivencia adecuada dentro de las instalaciones escolares. El presente estudio se basa en la situación de la violencia escolar en secundarias públicas, analizada desde la perspectiva del personal de apoyo (orientadores, psicólogos y prefectos) y la influencia que tiene en la percepción estudiantil del ambiente académico. El personal de apoyo tiene por objetivo contribuir al desarrollo integral del educando en su proceso de adaptación al medio ambiente escolar, social y económico (Secretaría de Educación Pública [SEP], 1981).

El profesiograma del personal de apoyo incluye a la trabajadora social, que tiene funciones de promoción, organización y desarrollo de grupos de alumnos, campañas y actividades para la formación integral del estudiantado; asesora, comunica y visita a los padres para el tratamiento de problemas relacionados con sus hijos, además, se coordina con la dirección del área de asistencia educativa; su perfil es Licenciado en Trabajo Social y Psicología (Subsecretaría de Educación Básica, 2014). El orientador educativo en el profesiograma tiene también categoría administrativa, y adicionalmente coordina a trabajadores sociales, médicos, docentes y asesores de grupo en los programas de acción dentro del plantel. También, junto con la dirección, organiza los programas de actividades escolares y extraescolares de proyección comunitaria que favorecen el desarrollo de los alumnos; su perfil es de psicología en todas sus especialidades (Subsecretaría de Educación Básica, 2014). El prefecto está encargado de "vigilar y orientar a los alumnos para que cumplan con responsabilidad y convencimiento, tanto con el reglamento escolar como con las disposiciones y medidas que señalen las autoridades y que desarrollen 
actitudes y hábitos que contribuyan a su formación integral" (SEP, 1981: 36). El trabajo que realizan los prefectos es diferente en cada escuela secundaria (Orozco, 2007; Tello, 2016), el cual depende del contexto, los alumnos y la forma en que cumplen su función; sin embargo, es este personal de apoyo el que se encarga de ejecutar los lineamientos, normas y reglamentos que la escuela negocia con los padres y los alumnos para mantener el orden, disciplina y generar identidad. Desde portar el uniforme hasta el largo de la falda, el cabello y denuncias de comportamiento agresivo dentro $y$ fuera de las aulas.

El trabajo que desempeña el personal de apoyo es muy importante y de gran relevancia para la solución de problemas dentro de la escuela ya que, al igual que los docentes, se mantienen frecuentemente en contacto con los estudiantes y llegan a representar modelos conductuales para ellos (James et al., 2008). Por lo que es importante formar y preparar al personal de apoyo en la resolución de problemas y conflictos (Fierro et al., 2013).

La necesidad de incrementar los estudios sobre violencia en las escuelas se debe a la complejidad del fenómeno y a las diferentes dinámicas que se presentan en contextos muy variados (Gómez, Zurita, 2013; Madrid et al., 2020). A través del análisis de la función de cada uno de los actores y su importancia en la prevalencia o solución del problema, se pueden obtener los elementos que ayuden a la comunidad escolar a lograr el objetivo principal: el cumplimiento de los aprendizajes esperados y el mantenimiento de la convivencia pacífica.

En el concurso de oposición para el ingreso a la educación básica del ciclo escolar 20152016, la Coordinación Nacional del Servicio Profesional Docente (CNSPD, 2017: 18), por motivo de la Ley General del Servicio Profesional, muestra un perfil con las características, cualidades y aptitudes deseables en los directivos de secundaria para poder desempeñar su labor profesional eficazmente. Este perfil se conforma por cinco dimensiones, la cuarta expresa: "Un director que asume y promueve los principios éticos y los fundamentos legales inherentes a su función y al trabajo educativo, con el fin de asegurar el derecho de los alumnos a una educación de calidad", lo cual es fundamental para desempeñar su labor. Entre los parámetros de esta dimensión, se menciona que los directivos deben reconocer la importancia de su intervención para asegurar la integridad de los estudiantes.

Por otro lado, la quinta dimensión describe a "Un director que reconoce el contexto social y cultural de la escuela y establece relaciones de colaboración con la comunidad, la zona escolar y otras instancias, para enriquecer la tarea educativa" (CNSPD, 2017: 19). Estas dos dimensiones se enfocan en las capacidades del directivo para identificar, valorar y aprovechar los elementos del entorno para establecer un óptimo clima escolar, donde se promueva la equidad, inclusión, respeto y empatía entre todos los actores del centro escolar, así como una relación de colaboración con el contexto local.

El apoyo directivo, como concepto y como ejercicio, incluye una gama de comportamientos que se pueden dividir en cuatro dimensiones (Littrell et al., 1994): apoyo emocional, apoyo 
instrumental, apoyo informacional y apoyo evaluativo. Las competencias, habilidades y actitudes del personal de apoyo hacia el liderazgo del director constituyen un soporte para que el colectivo docente se desarrolle profesionalmente, se sienta influido, dirigido, orientado y motivado en el logro de metas compartidas para conseguir una mejora permanente (Rincón, 2011).

Si bien es cierto que el director debe mostrar una imagen que represente autoridad, ésta debe complementarse con las habilidades del personal de apoyo que permitan hacer un manejo eficiente de las interacciones con su colectivo escolar, para lo cual, la comunicación constructiva y pacífica es una herramienta que permite la formación y el desarrollo del directivo, aprovechando la experiencia y conocimiento del colectivo escolar (Arzola et al., 2016).

Los conflictos son los desacuerdos o contraposiciones entre dos o más sujetos o grupos de personas (Rodríguez, 2008; Uruñuela, 2007) y se comprende que son por sí mismos inevitables y necesarios para consolidar las relaciones interpersonales en cualquier contexto (Quiroz, 2012). Sin embargo, la resolución de problemas requiere habilidades de enfrentamiento para que el conflicto promueva relaciones positivas en el grupo de pertenencia y no afecte el clima escolar negativamente, desencadenando en actos de violencia (Bautista et al., 2019).

Para Lazarus y Folkman (1986), el enfrentamiento comprende todos los esfuerzos cognitivos y conductuales cambiantes que las personas desarrollan para controlar las demandas específicas del ambiente - $\mathrm{y} / \mathrm{o}$ internas - que son percibidas como exuberantes o desbordantes de los medios de la persona. Los autores reconocen dos tipos de enfrentamiento, la distinción radica en la dirección del enfrentamiento. Cuando el enfrentamiento busca manipular la situación problema o alterarla, se le denomina enfrentamiento enfocado en el problema; cuando la dirección del enfrentamiento se enfoca en las respuestas emocionales que emergen del problema, se le llama enfrentamiento enfocado en las emociones, éste es posterior a evaluar la situación como inalterable (Lazarus, Folkman, 1986).

\section{Enfrentamiento y violencia escolar}

Si consideramos a la escuela como un agente socializador donde los alumnos aprenden normas, valores, relaciones interpersonales adecuadas, entre otros aspectos, la forma en que el personal de apoyo actúa ante los conflictos y problemas de interacción social que se presentan en los centros escolares convierte a este personal en un modelo de acción que los estudiantes intentarán imitar. Con base en esto, resulta muy importante que las prácticas de buena conducta y solución de problemas no sólo se promuevan de forma discursiva. El personal de apoyo tiene la obligación de comportarse de forma adecuada cuando estos se enfrentan a situaciones de violencia escolar (Chagas, 2005).

El perfil del personal de apoyo a la dirección no establece criterios e indicadores que enuncien la importancia del desarrollo de habilidades socioemocionales en los directivos y docentes, específicamente en las competencias sociales y de comunicación que corresponden a las 
dimensiones de liderazgo dentro de la escuela; el documento sólo considera estas habilidades en la formación del alumno y adjudica a la función del directivo la responsabilidad de crear espacios y ambientes donde se propicie la formación integral. "Las facultades de la inteligencia emocional son sinérgicas con las habilidades cognitivas; los trabajadores excelentes poseen los dos tipos. Cuanto más complejo es el trabajo, más importante es la inteligencia emocional" (Alarcón et al., 2017: 10). Si el personal de apoyo promueve y desarrolla en el directivo prácticas de liderazgo y acciones de gestión institucional, éstas impactan positivamente en la eficacia colectiva docente evidenciando que, a medida que el directivo inspira una visión en su colectivo, se asumen riesgos para modificar el curso de los acontecimientos desafiando el proceso (Sandoval et al., 2021).

Por otro lado, existe evidencia de que dependiendo del estilo de enfrentamiento en situaciones de violencia escolar entre pares y personal docente y directivo, disminuye o aumenta la victimización de los estudiantes; tal es el caso del estudio de Madrid et al. (2015), en donde mayores niveles de victimización se relacionan con un estilo evitativo emocional. Por el contrario, un estilo directo revalorativo se asocia a niveles bajos de victimización. Por lo tanto, se asume que enfrentar las situaciones de violencia escolar de una manera directa favorece interacciones menos agresivas.

\section{Clima escolar}

El clima escolar se relaciona con componentes positivos como la calidad de vida dentro de la institución académica; por ejemplo, una óptima interacción y cooperación basada en el respeto entre los integrantes de la comunidad escolar (profesorado, personal de apoyo y estudiantes), así como el deseo de crear un diálogo recíproco donde se compartan experiencias (Backhoff, Pérez, 2013).

La violencia escolar, como fenómeno complejo y multidimensional, requiere la atención de diversos factores para poder medirla. La investigación socioeducativa señala que los estudiantes tienden a participar con mayor frecuencia en situaciones de acoso escolar cuando el clima de la escuela es negativo. Esto pasa cuando los estudiantes perciben que en su ambiente escolar son normales los conflictos, la injusticia y la falta de apoyo. También se manifiesta en las conductas por parte de los estudiantes observadores que promueven que los agresores mantengan su acoso sobre las víctimas (Ferráns, Selman, 2014; Gendron et al., 2011).

Los estudiantes tienden a desarrollar actitudes de convivencia positiva cuando perciben que el personal de apoyo y los docentes los ayudan y los cuidan, creando así un clima escolar positivo (Low, Van Ryzin, 2014). Múltiples estudios dentro de las instituciones académicas han encontrado que cuando se favorece el respeto y la convivencia pacífica entre los integrantes de la comunidad escolar, y a la vez que se le brinda seguridad, existe una menor frecuencia de comportamientos violentos entre pares (Collie et al., 2011; Good et al., 2011; Waasdorp et al., 2012). 
Para el establecimiento de un clima escolar positivo es importante incorporar estrategias de intervención dentro del currículo escolar, además de hacer partícipe a toda la comunidad de esta intervención, y enfocarse en la construcción de relaciones positivas entre los diversos actores de la institución académica (Cohen, Freiberg, 2013; Wang et al., 2013). Por lo anterior, el objetivo del estudio es identificar la relación entre la percepción del clima escolar, el tipo de enfrentamiento por parte del personal de apoyo (prefectos, psicólogos y orientadores) y su percepción de violencia escolar en secundarias públicas del estado de Sonora, México.

\section{Método}

\section{Diseño y tipo de estudio}

El estudio tiene un enfoque cuantitativo con un diseño no experimental ex-post-facto de corte transversal y alcance explicativo (Abreu, 2012).

\section{Participantes}

Se utilizó un muestreo no probabilístico y por conglomerados, los cuales estaban constituidos por las escuelas que, según el Observatorio Ciudadano de Convivencia y Seguridad del Estado de Sonora (2017), se ubicaban en un sector con reportes e incidencias de violencia: violencia intrafamiliar, omisión de cuidados, negligencia, abuso físico, psicológico, sexual, drogadicción e incumplimiento de obligaciones. Para ello se analizaron estadísticas de incidencias de reportes de violencia, datos proporcionados por el mismo organismo; adicionalmente, los conglomerados deberían incluir escuelas de educación secundaria correspondientes al subsistema público, excluyendo a las de tipo telesecundaria y comunitaria. Participaron 154 personas que realizan trabajo de apoyo para la gestión directiva en 46 escuelas secundarias públicas de 14 municipios, ubicadas en cuatro zonas geográficas en Sonora: centro, frontera, sur y sierra; $46.6 \%$ de los participantes son del sexo masculino, con edades que oscilaron entre 24 y 68 (con un promedio de 42.64 años), y $53.4 \%$ del sexo femenino, con edades entre 19 y 58 años (con una media de 39.95 años). $17.7 \%$ se encuentran entre los 19 y los 30 años, $66 \%$ entre 31 y 50 años, y $16.3 \%$ es mayor de 51 años. $66.9 \%$ del total de los participantes laboran en el turno matutino y $33.1 \%$ en el vespertino.

\section{Instrumentos}

Escala de Percepción del Clima y del Funcionamiento del Centro (EPCFC). Se utilizó una adaptación de la EPCFC elaborada para profesores de España (Oliva et al., 2009). Solamente se tomaron 18 reactivos de los 22 que originalmente se propone la escala. La escala mide la calidad del centro respecto al clima escolar y su relación con el funcionamiento de la gestión directiva en la escuela desde la percepción del personal de apoyo. El instrumento tiene un coeficiente de fiabilidad para la escala en su conjunto (alfa de Cronbach $=.87$ ) y está dividido en seis dimensiones: 
Cohesión, Convivencia, Empoderamiento, Compromiso del personal de apoyo, Metas educativas y Vinculación, según los resultados del análisis factorial exploratorio (AFE) y explican $72 \%$ de la varianza total. Las respuestas se registran a partir de una escala tipo Likert de frecuencia de cinco puntos ( 1 = Nunca y $5=$ Siempre).

En este estudio, luego de realizar un AFE de máxima verosimilitud y rotación oblimin, se modificó la estructura de dos dimensiones $\left(\mathrm{KMO}=.69, X^{2}=95.8, p<.001\right)$. A la primera dimensión se le denominó Empoderamiento, que mide la percepción que tiene el personal de apoyo sobre la participación del alumnado para la organización, propuesta de actividades y normas de convivencia dentro del centro educativo (Peñalba et al., 2015). Esta dimensión está compuesta por tres ítems (por ejemplo:"Los estudiantes participan en la elaboración de las normas de convivencia de la escuela"). Obtuvo 31.06\% de la varianza y un alfa de Cronbach de .79. Todos los ítems seleccionados presentaban comunalidades mayores a .35 y pesos factoriales mayores a .40 .

A la segunda dimensión se le denominó Compromiso y vinculación, y mide la percepción del personal de apoyo acerca de su sentido de pertenencia con el centro educativo (Peñalba et al., 2015). Esta dimensión está compuesta de tres reactivos (por ejemplo: "Me siento a gusto en mi escuela"). Obtuvo $21.52 \%$ de la varianza y un alfa de Cronbach de .69. Se llevó a cabo después de un análisis factorial confirmatorio (AFC) para una muestra aleatoria de 100 funcionarios del personal de apoyo con las dos dimensiones, y los valores de ajuste fueron aceptables $\left(X^{2}(8)=\right.$ $16.84, p=.032 ; \mathrm{CFI}=.96 ; \mathrm{RMSEA}=.08, \mathrm{IC} 90[.02, .14] ; \mathrm{AGFI}=.90 ; \mathrm{SRMR}=.06 ; \mathrm{AIC}=42.84 ; \mathrm{BIC}=82.32)$.

Escala de Enfrentamiento ante la Violencia. Se utilizó una adaptación del cuestionario de Cassidy y Long (1996) sobre los estilos de resolución de conflictos. La escala se conforma de 24 ítems agrupados en seis estilos diferentes para la solución de problemas: Impotencia, Control, Creatividad, Confianza, Evitación y Enfoque. Las respuestas se registraron a partir de una escala tipo Likert de frecuencia de cinco puntos ( $1=$ Nunca y $5=$ Siempre).

En este sentido, se eligieron 18 del total de reactivos que se ajustaron a los estilos de solución relacionados con problemas y violencia entre estudiantes en las escuelas públicas por parte del personal de apoyo, sobre todo prefectos, trabajadora social y subdirectivos. Después de realizar un AFE de máxima verosimilitud con rotación oblimin, se mantuvieron dos dimensiones $\left(\mathrm{KMO}=.73, X^{2}=377.67, p<.001\right)$. La primera dimensión, denominada Enfrentamiento directo (control y confianza en la escala original): mide la percepción que tiene el personal de apoyo sobre la confianza en su capacidad para controlar los problemas de violencia entre los alumnos de la escuela (por ejemplo: "Puedo ver una salida a los problemas de violencia entre estudiantes y saber qué hacer"). Obtuvo $36.09 \%$ de la varianza y un alfa de Cronbach de .84 . La segunda dimensión, denominada Enfrentamiento evasivo: mide la percepción del personal de apoyo acerca de su esfuerzo cognitivo para evadir los problemas al considerar que el tiempo resolverá la violencia entre los estudiantes (por ejemplo: "Trato de ignorar u olvidar los problemas con los estudiantes"). Obtuvo $18.72 \%$ de la varianza y un alfa de Cronbach de .69 . 
Al igual que en el caso anterior, se utilizó una muestra aleatoria de 100 participantes para llevar a cabo un AFC para la escala obtenida en el exploratorio. Los valores de ajuste en el análisis confirmatorio con las dos dimensiones fueron aceptables $\left(\mathrm{X}^{2}(13)=21.86 ; p=.05 ; \mathrm{CFI}=.97\right.$; RMSEA= .06; IC $90[.00, .11] ; \mathrm{AGFI}=.92 ; \mathrm{SRMR}=.03 ; \mathrm{AIC}=51.86 ; \mathrm{BIC}=97.42)$.

Escala General de Violencia. Se trata de una medida que el personal respondía sobre la base de que la violencia es toda acción verbal o física que afecta los derechos de otras personas y les provoca emociones negativas. Se compone de cinco preguntas asociadas a los actores en díadas alumno-alumno, alumno-maestro, maestro-maestro, maestro-alumno y personal directivo-maestros. El nivel de violencia entre los actores se evalúa con una escala de respuesta que va de 1 (Muy bajo) a 5 (Muy alto). El valor de alfa de Cronbach es adecuado (.82) y el AFE de máxima verosimilitud y rotación oblimin presentó una escala unidimensional que explica $51 \%$ de la varianza total.

\section{Procedimiento}

Para recabar la información se obtuvo el consentimiento y colaboración del personal de apoyo de las instituciones. Se solicitó la participación voluntaria e informada del personal de apoyo a la gestión directiva en cada una de las escuelas seleccionadas para la muestra, garantizándoles la confidencialidad de la información. Previamente, se solicitaron permisos a los directivos para poder planear el horario de visita a las escuelas. Cabe señalar que la aplicación se realizó de manera individual, durante un tiempo aproximado de 30 minutos.

\section{Análisis estadísticos}

El análisis de los datos se realizó mediante análisis factorial confirmatorio, análisis de correlaciones de Pearson y análisis estructural (Hair et al., 2010). En el análisis estructural, donde se utilizó el método de estimación de máxima verosimilitud (ML), se evaluó a partir de diferentes índices de ajuste: $X^{2}$ y significancia estadística (> .05), AGFI (Bondad Ajustado de Índice de Ajuste) > .90, CFI (Índice de Ajuste Comparado) > .90, SRMR (Estandarizado Root Mean Residual) <.05 y RMSEA (Error de Aproximación Cuadrático Medio) < .05 (Manzano, Zamora, 2009) con su respectivo intervalo de confianza. Cabe señalar que, para sortear la distribución de los datos y que la normalidad multivariada no sea un problema, se utilizó el método bootstrap del AMOS, el cual consiste en 500 repeticiones y un intervalo de confianza de 95\% (Byrne, 2010); donde, además, los efectos indirectos se calcularon con el método de bootstrap, con las mismas repeticiones (500) e intervalo de confianza (95\%). Los análisis se realizaron con los paquetes estadísticos SPSS v22 y AMOS v23.

\section{Resultados}

En primer lugar, se realizaron análisis de correlación entre las dimensiones de enfrentamiento, clima escolar e índice general de violencia (Tabla 1). Se encontraron asociaciones negativas de 
bajas a medias, pero significativas entre el índice general de violencia y el enfrentamiento directo, el clima escolar referente al compromiso y vinculación con el centro escolar y referente al empoderamiento de los alumnos en las actividades escolares. La relación es positiva entre el índice general de violencia y el enfrentamiento evitativo.

Lo anterior indica que aquellos participantes que en sus centros de trabajo perciben la colaboración de los estudiantes en la elaboración de las normas de convivencia de la escuela, o bien, que se sienten a gusto en su escuela, perciben menor nivel de violencia entre los miembros del plantel educativo. La vinculación y compromiso que tiene el personal de apoyo con su institución tuvo mayor peso para percibir menor violencia que el empoderamiento de los alumnos.

Por otra parte, el personal de apoyo que se percibe a sí mismo como capaz de visualizar una salida a los problemas de violencia entre los estudiantes de la escuela y sabe qué hacer en estas situaciones, tiende a percibir menor nivel de violencia entre los miembros de su escuela, mientras que aquellos que tratan siempre de ignorar u olvidar los problemas con los estudiantes perciben mayor nivel de violencia.

Tabla 1. Correlaciones entre las variables de estudio

\begin{tabular}{|l|c|c|c|c|c|}
\hline & $\mathbf{1}$ & $\mathbf{2}$ & $\mathbf{3}$ & $\mathbf{4}$ & $\mathbf{5}$ \\
\hline 1. Índice General de Violencia & ---- & & & & \\
\hline 2. Enfrentamiento directo & $-.20^{*}$ & ----- & & & \\
\hline 3. Enfrentamiento Evitativo & $.22^{* *}$ & $-.20^{*}$ & ---- & & \\
\hline 4. Empoderamiento & $-.20^{*}$ & $.19^{*}$ & .09 & $-\cdots-$ & \\
\hline 5. Compromiso y vinculación & $-.35^{* *}$ & $.26 * *$ & $-.46 * *$ & .12 & $-\cdots$ \\
\hline
\end{tabular}

Nota. $p<.05^{*} . p<.01^{* *}$

\section{Modelo estructural}

A partir del desarrollo teórico y empírico reportado en la literatura y con los factores confirmados, se realizó un análisis estructural para estimar las relaciones entre los factores. En la figura 1 se presentan los coeficientes estandarizados que fueron significativos con nivel menor o igual a .01. Los índices de ajuste global del modelo teórico sugieren que se sustenta en el modelo de los datos empíricos $X^{2}(61)=80.314 ; X^{2} / g l=1.31, p=.04 ; \mathrm{CFI}=.97$, SRMR $=.02$, RMSEA $=.04$; IC 90\% [.003, .071]; AGFI .88). El modelo explica $25 \%\left(R^{2}\right)$ de la percepción de violencia entre los miembros del centro educativo.

De acuerdo con los coeficientes estandarizados y su probabilidad asociada, el enfrentamiento directo que percibe el personal de apoyo se relaciona positivamente con la dimensión de compromiso y vinculación ( $\beta=.21 ; p<.01$ ); mientras que el enfrentamiento evasivo, mediante el cual el personal de apoyo trata de ignorar los problemas entre los estudiantes, se asocia de 
manera negativa y con mayor fuerza con el compromiso y la vinculación que adquieren con su centro de trabajo $(\beta=-.62 ; p<.01)$. El compromiso y vinculación afecta disminuyendo la percepción del índice general de violencia $(\beta=.50 ; p<.01)$.

Figura 1. Resultados del cálculo del modelo estructural de las relaciones entre enfrentamiento, clima

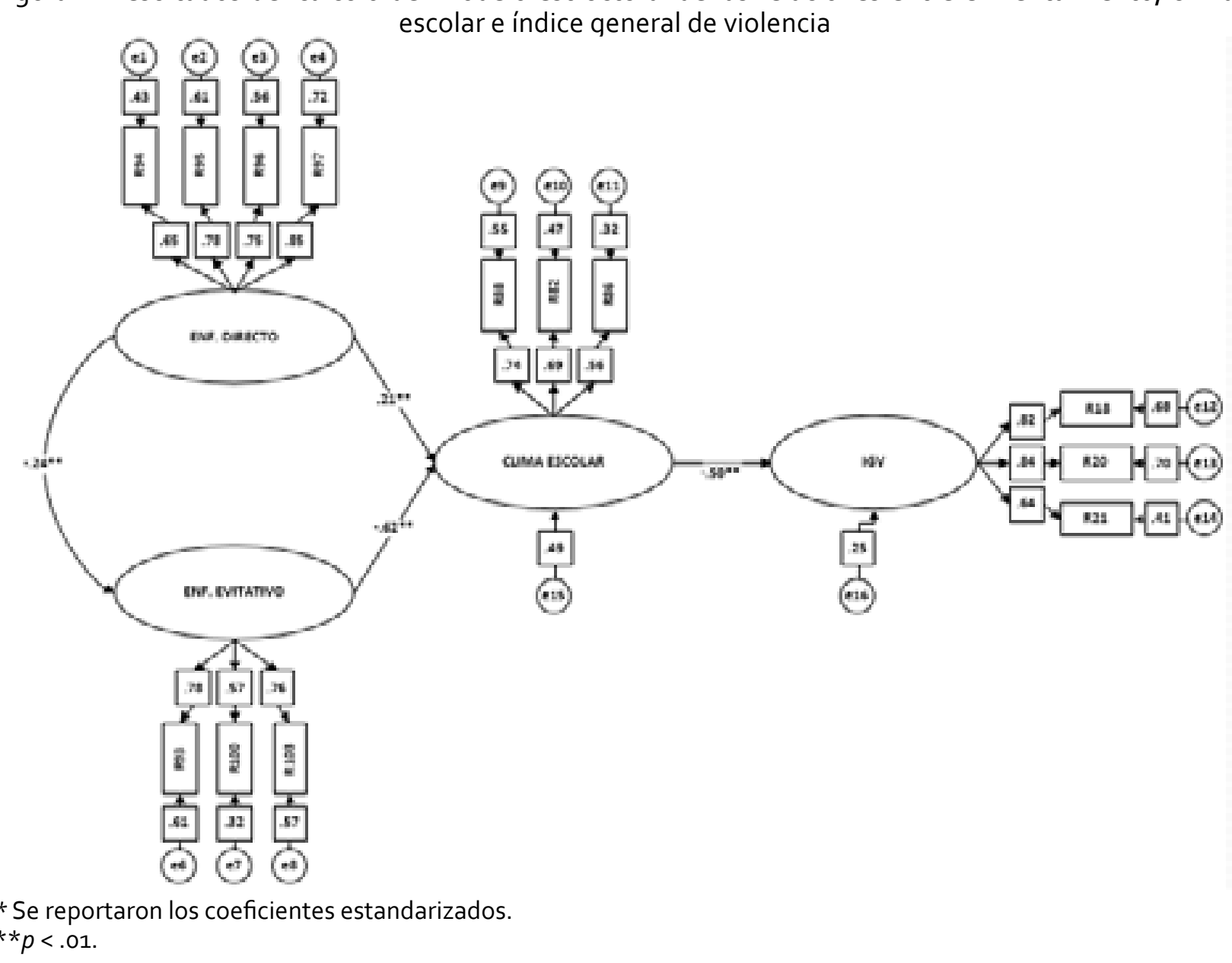

En lo referente a los efectos indirectos, se encontró que el enfrentamiento directo del conflicto disminuye la percepción del índice de violencia mediando su efecto con el clima escolar, es decir, con el compromiso y la vinculación que adquiere el personal de apoyo con su centro de trabajo ( $\beta=-.11 ; p<.05)$. Por el contrario, el enfrentamiento evasivo aumentaba la percepción de violencia en el ámbito escolar, mediando su efecto con el clima escolar (véase Tabla 2). 
Tabla 2. Relaciones indirectas entre enfrentamiento directo, enfrentamiento evasivo, clima escolar e índice general de violencia

\begin{tabular}{lccc}
\hline \multicolumn{1}{c}{ Relaciones indirectas } & $B$ & IC 95 & $p$ \\
\hline E. directo $\longrightarrow$ Clima escolar $\rightarrow$ IGV & -.11 & {$[-.26--.01]$} & .030 \\
E. evasivo $\longrightarrow$ Clima escolar $\rightarrow$ IGV & .31 & {$[.17-.47]$} & .001 \\
\hline
\end{tabular}

Nota. E. Directo = Enfrentamiento directo centrado en el problema, E. Evasivo = Enfrentamiento evasivo emocional, IGV = Índice General de Violencia.

\section{Discusión y conclusiones}

En este trabajo nos planteamos como objetivo identificar la relación entre la percepción del clima escolar, el tipo de enfrentamiento ante el conflico por parte del personal de apoyo (prefectos, psicólogos y orientadores) y la percepción de violencia escolar en secundarias públicas del estado de Sonora, México.

En lo que se refiere específicamente a la relación entre el clima escolar y la percepción de violencia escolar, nuestros resultados concuerdan con algunos estudios previos (Cerezo et al., 2015; Tijmes, 2012; Vera et al., 2021), en los cuales se señala que el clima escolar se relaciona de manera negativa con la percepción de violencia escolar.

Con respecto al tipo de enfrentamiento y la percepción de violencia, llama la atención la relación mayor que existe entre enfrentamiento evitativo y la percepción de violencia. Es decir, evitar enfrentar los episodios de violencia en la escuela tiene como consecuencia que el personal de apoyo perciba más violencia; pero percibirse con capacidad para enfrentarlos de manera directa disminuye la percepción de violencia. Esta relación es coherente con lo encontrado por Vera et al. (2021).

Estos hallazgos tienen pertinencia con el discurso sobre la naturalización de la violencia que con frecuencia encontramos en la literatura (Galán, 2018; Pacheco-Salazar, 2018). Se dice que la naturalización está presente en el ámbito educativo, dado que se trata de uno de los espacios a los que se extiende la violencia estructural del país (Cano, Estrada, 2015; Conde, 2014); pero poco se ha reflexionado sobre las habilidades de enfrentamiento que tienen las personas, en este caso, el personal de apoyo, para hacer frente a la violencia que se presenta en su centro escolar.

Lo anterior se reafirma con los resultados del análisis estructural, específicamente cuando los resultados señalan claramente cómo aquellos participantes que tratan de ignorar la problemática de violencia perciben un menor compromiso y vinculación con el centro, mientras que los participantes con enfrentamiento directo tienden a percibir mayor compromiso y vinculación con el centro.

En un contexto en el que suele describirse al personal de apoyo directivo de las escuelas secundarias como de origen social con características de la cultura del control y del poder, ma- 
nejan un concepto de sobrevivencia darwiniano basado en las habilidades del más fuerte (Tello, 2013), existe una imperante necesidad de entrenamiento y capacitación para el enfrentamiento directo de la agresión y la violencia entre pares, para desnaturalizar las conductas antisociales, disminuir la apatía y la falta de atención hacia las conductas que promueven una escuela insegura y violenta (Ferráns, Selman, 2014).

Dados los vacíos de conocimiento existentes en torno al papel del personal de apoyo dentro de las escuelas frente a la violencia escolar, este estudio representa un esfuerzo para encontrar las rutas y opciones frente a una problemática que se presenta como compleja y con varias aristas. Algunas limitantes a la validez externa (Kerlinger, Lee, 2002) están relacionadas con la selección de personal de apoyo directivo de escuelas secundarias en polígonos de violencia (Observatorio Ciudadano de Convivencia y Seguridad del Estado de Sonora, 2017). Adicionalmente, en las escuelas secundarias existe una trabajadora social, hasta tres prefectos, y en pocas ocasiones un orientador educativo, por lo cual, el número de cada uno de ellos no está igualado. Lo anterior implica que el número de repeticiones de cada actor es desigual dentro del total de la muestra. Para la validez interna tenemos la falta de aleatorización y grupos de comparación, además de que tenemos sólo un corte transversal en tiempo (McMillan, Schumacher, 2005).

Futuras investigaciones deberán profundizar en el manejo de la desconexión moral por parte del personal directivo y de apoyo, como antecedente de la naturalización de la violencia (Hymel et al., 2005). Por otro lado, identificar los parámetros relacionados con la eficacia colectiva docente, que ha mostrado constituirse en un factor fundamental para disminuir la violencia en la escuela y está vinculado a las labores de gestión directiva (Calik et al., 2012). Finalmente, los riesgos psicosociales derivados de la labor magisterial tendrán que ser examinados para determinar su impacto sobre la percepción de eficacia y capacidad de enfrentamiento de la violencia escolar (Littrell et al., 1994).

\section{Referencias}

Abreu, J. (2012). Hipótesis, método y diseño de investigación (Hypothesis, Method \& Research Design). Daena: International Journal of Good Conscience, 7(2), 187-197.

Alarcón, D.; R. Fuentes; H. Armendáriz (2017). La inteligencia emocional y su relación con el liderazgo de los directores de escuelas de educación básica. XIV Congreso Nacional de Investigación Educativa. San Luis Potosí, México: COMIE.

Arzola, D.; C. Loya; A. González (2016). El trabajo directivo en educación primaria: liderazgo, procesos participativos y democracia escolar. IE Revista de Investigación Educativa de la REDIECH, 7(12), 35-41. https://www.redalyc.org/articulo.oa?id=521653208004 
Backhoff, E.; J. Pérez (coords.) (2013). Segundo Estudio Internacional sobre la Enseñanza y el Aprendizaje (TALIS 2013). Resultados de México. México: INEE.

Byrne, B. (2010). Structural Equation Modeling with AMOS. Basic Concepts, Applications, and Programming. Nueva York: Routledge.

Bautista, G.; A. Vera; J.Tánori (2019). Shame, Empathy, Coping and School Safety of the Bystanders in Situations of Bullying. Trends in Psychology, 27(2), 357-369. https://doi.org/10.9788/ tp2019.2-05

Cano, A; M. Estrada (2015). Violencia estructural y estudiantes de escuelas secundarias del noroeste fronterizo de Chihuahua, México. Revista Eleuthera, (12), 34-55. https://revistasojs.ucaldas.edu.co/index.php/eleuthera/article/view/3743

Calik, T.; F. Sezgin; H. Kavgaci; A. Kilinc (2012). Examination of Relationships Between Instructional Leadership of School Principals and Self-efficacy of Teachers and Collective Teacher Efficacy. Educational Sciences: Theory and Practice, 12(4), 2498-2504. https://eric.ed.gov /?id=EJ1002859

Cassidy T.; C. Long (1996). Problem-solving Style, Stress and Psychological Illness: Development of a Multifactorial Measure. The British Journal of Clinical Psychology 35(2), 265-277. https://doi.org/10.1111/j.2044-8260.1996.tb01181.x

Cerezo, F.; E. Prodócimo; A. Caetano; M. Brighenti (2015). El clima escolar: percepción de la violencia y de la seguridad escolar por los estudiantes. XV Jornadas Internacionales Psicología Educacional. Chile: Universidad de Santiago de Chile.

Chagas, D. (2005). Los maestros frente a la violencia entre alumnos. Revista Mexicana de Investigación Educativa, 10(27), 1071-1082. https://www.redalyc.org/pdf/140/14002706.pdf

Cohen, J.; A. Freiberg (2013). School Climate and Bullying Prevention. En Dary, T.; T. Pickeral (eds.). School Climate Practices for Implementation and Sustainability. A School Climate Practice Brief, (1), 47-51. National School Climate Center. https://www.issuelab.org/resources/15024/15024.pdf

Collie, R.; J. Shapka; N. Perry (2011). Predicting Teacher Commitment: The Impact of School Climate and Social-emotional Learning. Psychology in the Schools, 48(10), 1034-1048. https:// doi.org/10.1002/pits.20611

Conde, S. (2014). La violencia y la cultura de la calle entran a la escuela: acciones y reacciones. Sinéctica, (42). https://sinectica.iteso.mx/index.php/SINECTICA/article/view/63

Coordinación Nacional del Servicio Profesional Docente (2017). Perfil, parámetros e indicadores para docentes y técnicos docentes. México: SEP. https://www.gob.mx/cms/uploads/attachment/file/264260/Perfil para metros e indicadores para personal con funciones de direccio $n$ y de supervisio $n$ EB .pdf

Ferráns, S.; R. Selman (2014). How Students' Perceptions of the School Climate Influence their Choice to Upstand, Bystand, or Join Perpetrators of Bullying. Harvard Educational Review, 84(2), 162-187. https://doi.org/10.17763/haer.84.2.h4883134101651mm 
Fierro, M.; A. Lizardi; G. Tapia; M. Juárez (2013). Convivencia escolar: Un tema emergente de investigación educativa en México. En Furlán, A.; T. Spitzer (coords.). Convivencia, disciplina y violencia en las escuelas. México: ANUIES/COMIE, 71-132.

Galán, J. (2018). Exposición a la violencia en adolescentes: desensibilización, legitimación y naturalización. Diversitas, 14(1), 55-67. https://doi.org/10.15332/s1794-9998.2018.0001.04

Gendron, B.; K. Williams; N. Guerra (2011). An Analysis of Bullying among Students within Schools: Estimating the Effects of Individual Normative Beliefs, Self-esteem, and School Climate. Journal of School Violence, (10), 150-164. https://doi.org/10.1080/15388220.2010.539166

Gómez, A.; Ú. Zurita (2013). El estudio de la violencia escolar, orígenes y tendencias. En Furlán, A.; T. Spitzer (coords.). Convivencia, disciplina y violencia en las escuelas. México: ANUIES/ COMIE, 183-222.

Good, C.; K. McIntosh; C. Gietz (2011). Integrating Bullying Prevention into Schoolwide Positive Behavior Support. Teaching Exceptional Children, 44(1), 48-56. https://doi. org/10.1177/004005991104400106

Hair, J.; W. Black; B. Babin; R. Anderson (2010). Multivariate Data Analysis (7ma. ed.). Nueva Jersey: Prentice Hall.

Hymel, S., N. Rocke-Henderson; R. Bonnano (2005). Moral Disengagement: A Framework for Understanding Bullying among Adolescents. Journal of Social Sciences, (8), 33-43.

James, D.; M. Lawlor; P. Courtney; A. Flynn; B. Henry; N. Murphy (2008). Bullying Behavior in Secondary Schools: What Roles do Teachers Play? Child Abuse Review, 17(3), 160-173. https://doi.org/10.1002/car.1025

Kerlinger, F.; H. Lee (2002). Investigación del comportamiento. México: McGraw-Hill.

Lazarus, R.; S. Folkman (1986). Estrés y procesos cognitivos. Evaluación, afrontamiento y consecuencias adaptativas. Nueva York: Roca.

Littrell, P.; B. Billingsley; H. Lawrence (1994). The Effects of Principal Support on Special and General Educator's Stress, Job Satisfaction, School Commitment, Health and Intent to Stay in Teaching. Remedial and Special Education, 15(5), 297-310. https://doi. org/10.1177/074193259401500505

Low, S.; M. Van Ryzin (2014). The Moderating Effects of School Climate on Bullying Prevention Efforts. School Psychology Quarterly, 29(3), 306-319. https://doi.org/10.1037/spq0000073

Madrid, E.; A. Valdés; M. Urías; G. Torres; L. Parra (2020). Factores asociados al ciberacoso en adolescentes. Una perspectiva ecológico-social. Perfiles Educativos, 42(167), 68-83. https://doi. org/10.22201/iisue.24486167e.2019.167.59128

Madrid, E.; A. Valdés; J. Vera (2015). Diferencias en los estilos de afrontamiento de estudiantes y víctimas y no involucrados en el bullying. XIII Congreso Nacional de Investigación Educativa. Chihuahua, México: COMIE.

Manzano, A.; S. Zamora (2009). Sistema de ecuaciones estructurales: una herramienta de investigación. Cuaderno técnico. México: Centro Nacional de Evaluación para la Educación Superior. 
McMillan, J.; S. Schumacher (2005). Investigación educativa (5ta. ed.). España: Pearson Educación. Observatorio Ciudadano de Convivencia y Seguridad del Estado de Sonora (2017). Análisis comparativo de incidencia delictiva. http://www.observatoriodesonora.org/que.html

Oliva, A.; L. Antolín; M. Pertegal; M. Ríos; A. Parra; A. Hernando; M. Reina (2009). Escala de percepción del clima y del funcionamiento del centro (profesorado). En Oliva, A.; L. Antolín; M. Pertegal; M. Ríos; A. Parra; A. Hernando; M. Reina (eds.). Instrumentos para la evaluación del desarrollo positivo adolescente y los activos que lo promueven. Universidad de Sevilla, 259-275.

Orozco, A. (2007). Disciplina y convivencia en escuelas secundarias. Un estudio cualitativo. IX Congreso Nacional de Investigación Educativa. Mérida, Yucatán, México: COMIE.

Pacheco-Salazar, B. (2018). Violencia escolar: la perspectiva de estudiantes y docentes. Revista Electrónica de Investigación Educativa, 20(1), 112-121. https://doi.org/10.24320/redie.2018.20.1.1523

Peñalba, V.; G. López; O. Vega; A. Satrustregui (2015). Clima escolar y percepciones del profesorado tras la implementación de un programa de convivencia escolar. Estudios sobre Educación, 28, 9-28. https://doi.org/10.15581/004.28.9-28

Quiroz, J. (2012). Violencia y conflictos escolares: definiciones conceptuales para la construcción de una nueva cultura de la legalidad. Rayuela, 5, 175-185.

Rincón, L. (2011). Liderazgo orientado a la competitividad y el desarrollo humano. Tesis de maestría. Universidad Nacional Abierta y a Distancia, Colombia.

Rodríguez, J. (2008). Los docentes ante las situaciones de violencia escolar. Revista Electrónica Interuniversitaria de Formación del Profesorado, 11(3), 32-39.

Sandoval, A.; P. Encinas; Y. Gutiérrez; J. Vera (2021). Liderazgo directivo: su relación con apoyo institucional y eficacia colectiva ante situaciones de conflicto. Revista Caribeña de Ciencias Sociales, 10(7), 69-84. https://doi.org/10.51896/caribe/MUTX4993

Secretaría de Educación Pública (1981). Manual de organización de la escuela de la educación secundaria. México: SEP. https://es.slideshare.net/faltoyano/manual-de-organizacin-de-laescuela-secundaria-26434259

Subsecretaría de Educación Básica (2014). Profesiograma, personal de asistencia educativa. MéxiCO: SEP. http://servicioprofesionaldocente.sep.gob.mx/portal-docente-2014-2018/content/ ba/docs/2015/permanencia/parametros indicadores/PPI DESEMPENO DOCENTE TECDOCENTES.pdf

Tello, N. (2013). La violencia escolar como forma de convivencia de una sociedad. Trabajo Social UNAM, (4), 43-56. http://revistas.unam.mx/index.php/ents/article/view/54050

Tello, N. (2016). El cambio en Trabajo Social: intención, rupturas y estrategias. México. En Cano, L.; E. Pastor (coords.). Políticas e intervenciones ante los procesos de vulnerabilidad y exclusión de personas y territorios. Análisis comparado. Ciudad de México: UNAM- Escuela Nacional de Trabajo Social/Dykinson, 89-101. 
Tijmes, C. (2012). Violencia y clima escolar en establecimientos educacionales en contextos de alta vulnerabilidad social de Santiago de Chile. Psykhe, 21(2), 105-117.

Uruñuela, P. (2007). Convivencia y conflictividad en las aulas. Análisis conceptual. En Moreno, A. (ed.), La disrupción en las aulas. Problemas y soluciones. Ministerio de Educación y Ciencia, 17-45.

Vera, J.; J. Aguayo; F. Favela; B. Parra (2021). Eficacia colectiva y gestión directiva y su relación con el clima y la violencia escolar. En Ramos, M.; L. Ramos; E. Noriega (eds.). Redes de cultura escolaren pro de la educación para la paz. Experiencias, análisis y propuestas de intervención. México: Fundación Vivir en Armonía, 333-342. https://www.researchgate.net/publication/352197232 Eficacia colectiva y gestion directiva y su relacion con el clima y la violencia escolar

Waasdorp, T.; C. Bradshaw; P. Leaf (2012). The Impact of Schoolwide Positive Behavioral Interventions and Supports on Bullying and Peer Rejection: A Randomized Controlled Effectiveness Trial. Archives of Pediatrics \& Adolescent Medicine, 166(2), 149-156. https://doi.org/10.1001/ archpediatrics.2011.755

Wang, C.; B. Berry; S. Swearer (2013). The Critical Role of School Climate in Effective Bullying Prevention. Theory into Practice, 52(4), 296-302. https://doi.org/10.1080/00405841.2013.829735 